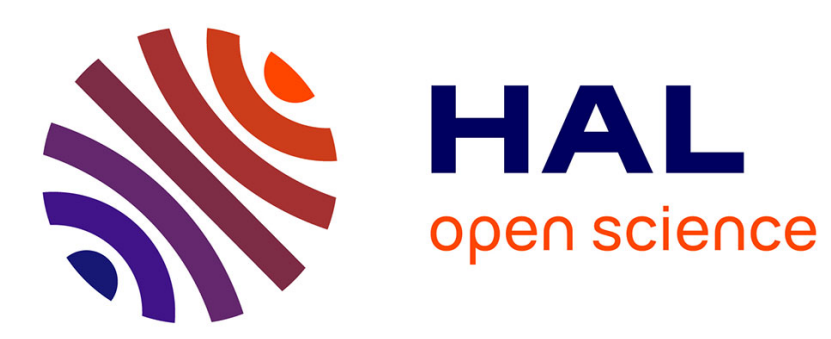

\title{
Brittle Fracture Theory Predicts the Equation of Motion of Frictional Rupture Fronts
}

Ilya Svetlizky, David S Kammer, Elsa Bayart, Gil Cohen, Jay Fineberg

\section{To cite this version:}

Ilya Svetlizky, David S Kammer, Elsa Bayart, Gil Cohen, Jay Fineberg. Brittle Fracture Theory Predicts the Equation of Motion of Frictional Rupture Fronts. Physical Review Letters, 2017, 118 (12), 10.1103/physrevlett.118.125501 . hal-03025965

\section{HAL Id: hal-03025965 https://hal.science/hal-03025965}

Submitted on 26 Nov 2020

HAL is a multi-disciplinary open access archive for the deposit and dissemination of scientific research documents, whether they are published or not. The documents may come from teaching and research institutions in France or abroad, or from public or private research centers.
L'archive ouverte pluridisciplinaire HAL, est destinée au dépôt et à la diffusion de documents scientifiques de niveau recherche, publiés ou non, émanant des établissements d'enseignement et de recherche français ou étrangers, des laboratoires publics ou privés. 


\title{
Brittle Fracture Theory Predicts the Equation of Motion of Frictional Rupture Fronts
}

\author{
Ilya Svetlizky, ${ }^{1}$ David S. Kammer, ${ }^{2}$ Elsa Bayart, ${ }^{1}$ Gil Cohen, ${ }^{1}$ and Jay Fineberg, ${ }^{1, *}$ \\ ${ }^{1}$ The Racah Institute of Physics, The Hebrew University of Jerusalem, Jerusalem 91904, Israel \\ ${ }^{2}$ School of Civil and Environmental Engineering, Cornell University, Ithaca, New York 14853, USA
}

(Received 2 November 2016; published 21 March 2017)

\begin{abstract}
We study rupture fronts propagating along the interface separating two bodies at the onset of frictional motion via high-temporal-resolution measurements of the real contact area and strain fields. The strain measurements provide the energy flux and dissipation at the rupture tips. We show that the classical equation of motion for brittle shear cracks, derived by balancing these quantities, well describes the velocity evolution of frictional ruptures. Our results demonstrate the extensive applicability of the dynamic brittle fracture theory to friction.
\end{abstract}

DOI: 10.1103/PhysRevLett.118.125501

A system of two bodies in frictional contact, subject to shear loading, is prone to lose stability and generate frictional slip. The onset of this motion was shown to be mediated by dynamically propagating fronts that rupture the discrete contacts forming the frictional interface [1] and separate the sticking and sliding regions [2-4]. A wide range of rupture front velocities $C_{f}$ have been observed. These span from slow ruptures $[1,3,5,6]$ propagating at a small fraction of the Rayleigh wave speed, $C_{R}$, to ruptures that asymptotically approach $C_{R}$ [7]. Rupture fronts may also surpass the shear wave speed, $C_{S}$, and reach the longitudinal speed of sound, $C_{L}[4,5,8,9]$.

Experiments [5,9] and simulations [10,11] have revealed that rupture fronts increase their speed with increased shear to normal stress ratios. Slow ruptures have received special attention due to the recent observation of slow earthquakes [12], and nontrivial constitutive laws of friction have been invoked to explain their existence and stability [11,13-15].

Further experiments have shown that the elastic fields in the tip vicinity of a frictional rupture are very well described by the universal singular solutions obtained by linear elastic fracture mechanics (LEFM), originally developed to describe brittle shear cracks. This was shown for ruptures propagating at $C_{f}<C_{R}$ along dry $[4,7,16]$ as well as boundary-lubricated interfaces [17]. In the LEFM framework, crack propagation velocities are determined by the balance between the energy flow to the crack tip and the dissipated energy per unit area, $\Gamma[18]$. This "energy balance" was successfully employed to predict the arrest of frictional rupture fronts $[19,20]$.

In this Letter, we experimentally investigate the dynamic propagation of frictional ruptures below $C_{R}$. The measurements will show that the velocity and the acceleration of these ruptures are described by the classical LEFM-based equation of motion for shear cracks in a brittle material. These experimental observations demonstrate the intimate relation between friction and fracture.

The experiments are conducted by using two poly(methylmethacrylate) (PMMA) plates $\left(\rho \approx 1170 \mathrm{~kg} / \mathrm{m}^{3}\right)$ having the same thickness. Rayleigh, shear, and longitudinal wave speeds are, respectively, $C_{R} \approx 1237 \mathrm{~m} / \mathrm{s}, C_{S} \approx 1345 \mathrm{~m} / \mathrm{s}$, and $C_{L} \approx 2333 \mathrm{~m} / \mathrm{s}$ (plane stress) [16]. The contacting surfaces were cleaned by isopropyl alcohol and dried for about $2 \mathrm{~h}$ (dry interfaces from here on). We also conduct experiments in the boundary-lubrication regime where contacting surfaces were coated by a thin layer of lubricant (silicon oil with kinematic viscosity $\nu \sim 100 \mathrm{~mm}^{2} / \mathrm{s}$ ). The two plates are carefully aligned and pressed together by an external normal force, $F_{N} \approx 5500 N$ ( $5 \mathrm{MPa}$ of nominal pressure) [see Fig. 1(a)]. Afterwards, shear forces $F_{S}$ are applied quasistatically until the desired values of elastic energies are imposed. Slip events are subsequently nucleated on demand by inducing a slight out of plane shear (mode III) perturbation at $x \approx 0$. The imposed quasistatic force perturbations in the $z$ direction [green arrows in Fig. 1(a)] were generally 2-3 orders of magnitude below $F_{S}$. During an event, a high-speed camera (580 000 frames/s) was used to visualize the dynamic changes in the real area of contact, $A(x, t)$. Simultaneously, the three components of the 2D strain tensor, $\varepsilon_{i j}(x, t)$, are continuously measured $\sim 3.5 \mathrm{~mm}$ above the frictional interface, at multiple locations along the plate each $1 \mu \mathrm{s}$. $\varepsilon_{i j}(x, t)$ measurements at discrete locations are converted to spatial profiles by taking advantage of the high temporal resolution, $\varepsilon_{i j}(x, t)=$ $\varepsilon_{i j}\left(x-\int C_{f} d t\right)$ [7]. Stresses $\sigma_{i j}$ are calculated from the measured $\varepsilon_{i j}$ by using the viscoelastic constitutive law of PMMA [19].

Figure 1(b) presents a typical measurement of $A(x, t)$. As the rupture propagates, the real area of contact is reduced, with the position of the boundary between regions of intact and reduced area of contact defining the rupture tip position $x_{\text {tip }}$. The rupture nucleates at $x \approx 0$ and accelerates in the positive $x$ direction, with $C_{f}$ asymptotically approaching $C_{R}$. The related shear stress $\sigma_{x y}$ for $x_{\text {tip }}=105 \mathrm{~mm}$ is plotted in Fig. 1(c) (green line). $\sigma_{x y}^{0}$ denotes the initially imposed shear. Residual stresses $\sigma_{x y}^{r}$ are measured at times corresponding to $x-x_{\text {tip }}=-40 \mathrm{~mm}$, well beyond any 
(a)

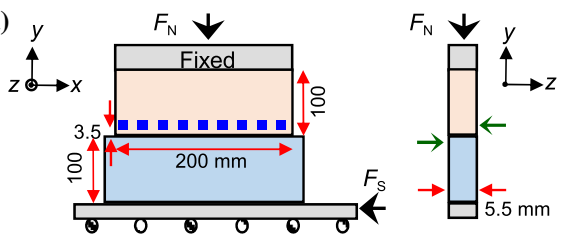

(b)

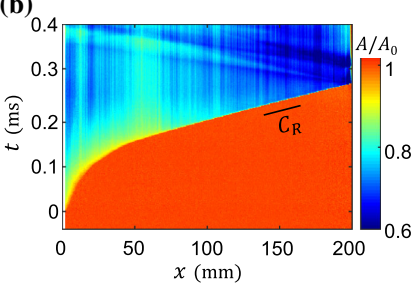

(c)

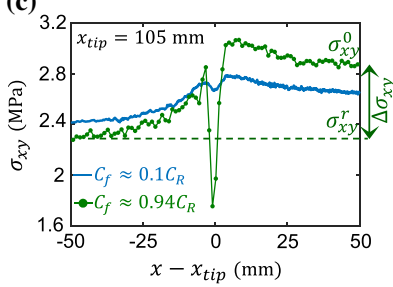

FIG. 1. The experimental system and definition of the dynamic stress drop. (a) Two identical PMMA blocks are used in a stick slip friction experiment. The elastic medium is considered to be $2 \mathrm{D}$ with a quasi-1D frictional interface, as the $5.5 \mathrm{~mm}$ width of the blocks is smaller than any other system dimensions. Full 2D tensorial strains are measured every $1 \mu \mathrm{s}$ at $16-19$ locations by miniature Rosette strain gauges mounted $\sim 3.5 \mathrm{~mm}$ above the interface (blue squares). Slip events are nucleated by applying minute out of plane perturbations at $x \approx 0$ (green arrows). (b) The evolution of the real area of contact, $A(x, t)$, for a typical rupture front propagating along dry interface (see the text). Nucleating at $x \approx 0$, the front rapidly accelerates towards the Rayleigh wave speed, $C_{R} \approx 1237 \mathrm{~m} / \mathrm{s}$, leaving in its wake a reduced area of contact. (c) Shear stresses $\sigma_{x y}$ plotted relative to the rupture tip position $x_{\text {tip }}=105 \mathrm{~mm}$. Green line: Rupture event in (b), propagating with the local rupture velocity $C_{f} \approx 0.95 C_{R}$. Blue line: A slow rupture propagating at $C_{f} \approx 0.1 C_{R} . \sigma_{x y}^{0}$ is the initial stress level, prior to the rupture arrival, while $\sigma_{x y}^{r}$ denotes the residual stress measured at times corresponding to $x-x_{\text {tip }}=-40 \mathrm{~mm}$. Their difference $\Delta \sigma_{x y}=\sigma_{x y}^{0}-\sigma_{x}^{r}$ defines the dynamic stress drop. Smaller $\Delta \sigma_{x y}$ accompany slower ruptures.

dynamic stress variation associated with the rupture tip. $\sigma_{x y}^{r}$, therefore, reflects the frictional resistance during sliding due to the nonzero residual value of $A(x, t)$ after the rupture passage [Fig. 1(b)]. The difference $\Delta \sigma_{x y}=\sigma_{x y}^{0}-\sigma_{x y}^{r}$, sometimes referred as the dynamic stress drop, plays a crucial role in the rupture dynamics, as it provides a measure for the amount of released elastic energy. Figure 1(c) (blue line) shows an additional example of a slow rupture $\left(C_{f} \approx 0.1 C_{R}\right)$, having a smaller $\Delta \sigma_{x y}$. Note that $\sigma_{x y}^{r}$ slightly decreases with an increased rupture velocity.

Three distinct profiles of $\Delta \sigma_{x y}(l)$ and $C_{f}(l)$ are plotted in Figs. 2(a) and 2(b), respectively. $l$ denotes the rupture length (as ruptures are nucleated at $x \approx 0, l=x_{\text {tip }}$ and $\left.C_{f}=\dot{l}\right)$. Lower values of $\Delta \sigma_{x y}$ are associated with slower ruptures, while the $C_{f}$ accelerates towards $C_{R}$ more rapidly when $\Delta \sigma_{x y}$ is higher. Different $\Delta \sigma_{x y}(l)$ profiles result from our controlled rupture initiation procedure; the system was first sheared to an initial stress level $\sigma_{x y}^{0}(x)$, and rupture nucleation was precipitated by applying a small out of
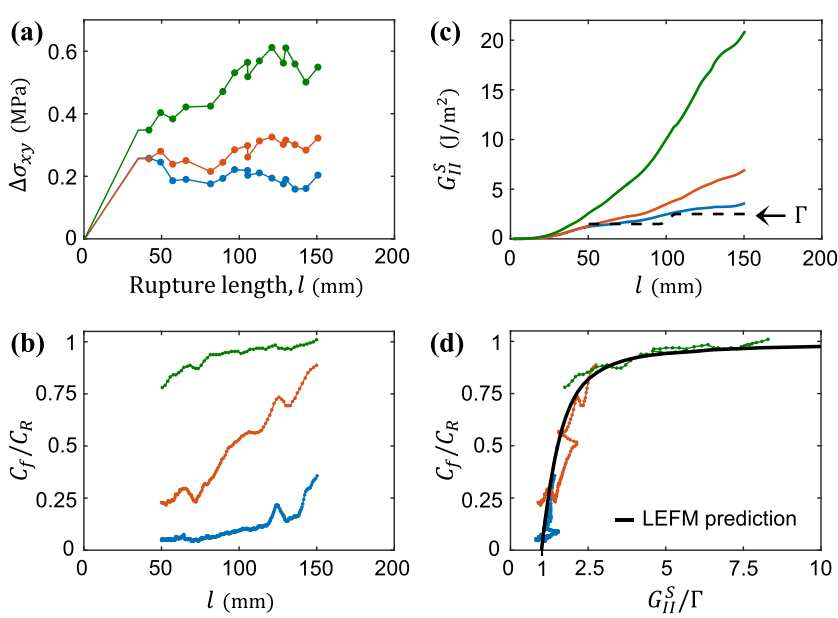

FIG. 2. Determining rupture velocity scaling. Colors represent the analysis of three rupture events that include the examples in Fig. 1(c). l: Rupture length. (a) Solid dots are measured $\Delta \sigma_{x y}$. $\Delta \sigma_{x y}$ near $x=0$ are extrapolated to $\Delta \sigma_{x y}=0$ at $x=0$ [21]. (b) Measured rupture velocity profiles $C_{f}(l)$. (c) The profiles of $\Delta \sigma_{x y}$ yield static energy release rates $G_{\mathrm{II}}^{S}\left(l, \Delta \sigma_{x y}\right)$ (see Ref. [21] for details). Dashed line: The measured fracture energy profile $\Gamma$. Slow rupture fronts (blue line) are associated with $G_{\text {II }}^{S} \approx \Gamma$, while ruptures rapidly accelerate towards $C_{R}$ (green line) once $G_{\text {II }}^{S} \gg \Gamma$. (d) $C_{f}(l)$ profiles collapse to a single functional form $C_{f}\left(G_{\mathrm{II}}^{S} / \Gamma\right)$, given by (black line) the classical equation of motion for shear cracks, Eq. (2).

plane (mode III) shear stress perturbation [Fig. 1(a)]. By controlling the nucleation, we were able to systematically change the initial stress state of the interface and generate large variations of rupture velocity profiles. This is in contrast to Refs. [5,7], where large $C_{f}$ variations resulted from uncontrolled spontaneous rupture nucleation. Our results, however, are independent of the nucleation procedure.

How does LEFM predict $C_{f}(l)$ ? LEFM predicts that the stress fields at the tip of a propagating shear crack (mode II) are described by the universal singular form

$$
\sigma_{i j}(\theta, r)=\frac{K_{\mathrm{II}}}{\sqrt{2 \pi r}} \Sigma_{i j}^{\mathrm{II}}\left(\theta, C_{f}\right),
$$

where $\Sigma_{i j}^{\mathrm{II}}\left(\theta, C_{f}\right)$ is a known universal function and $K_{\mathrm{II}}$ is known as the stress intensity factor. Recent work [7,16,17] has shown that precisely this singular form describes the fields at the tip of frictional ruptures. LEFM relates $K_{\text {II }}$ to the energy flow to the crack tip per unit area (known as the energy release rate), $G_{\mathrm{II}}=K_{\mathrm{II}}^{2} f_{\mathrm{II}}\left(C_{f}\right) / E$, where $f_{\mathrm{II}}\left(C_{f}\right)$ is a known universal function and $E$ is the Young's modulus [18]. For a crack to propagate energy balance, $\Gamma=G_{\mathrm{II}}$ should hold.

For a shear crack subjected to time-independent loading in an infinite medium, $G_{\mathrm{II}}=G_{\mathrm{II}}^{S}\left(l, \Delta \sigma_{x y}\right) g_{\mathrm{II}}\left(C_{f}\right)$, where all information about the loading is incorporated in $G_{\mathrm{II}}^{S}\left(l, \Delta \sigma_{x y}\right)$, the static energy release rate. $G_{\mathrm{II}}^{S}$ is a weighted 
integral functional of $\Delta \sigma_{x y}(x)$ which can be explicitly calculated, if the $\Delta \sigma_{x y}(x)$ are known [21]. For example, for homogeneous loading, where $\Delta \sigma_{x y}(x)$ is independent of $x, G_{\mathrm{II}}^{S}$ is an increasing function of the crack length: $G_{\mathrm{II}}^{S} \propto\left(\Delta \sigma_{x y}\right)^{2} l$. The dynamic correction to the energy flux is given by $g_{\mathrm{II}}\left(C_{f}\right)$, a universal monotonic function that is unity at the limit $C_{f} \rightarrow 0$ and zero at $C_{f}=C_{R}$. Under these conditions, the equation for crack motion for arbitrary stress configurations is implicitly given by

$$
\Gamma=G_{\mathrm{II}}=G_{\mathrm{II}}^{S}\left(l, \Delta \sigma_{x y}\right) g_{\mathrm{II}}\left(C_{f}\right) .
$$

The finite value of $\Gamma$ together with $g_{\mathrm{II}}\left(C_{f}\right) \rightarrow 0$ as $C_{f} \rightarrow C_{R}$ imply that the limiting crack velocity $C_{R}$ can be reached asymptotically only when $G_{\mathrm{II}}^{S}\left(l, \Delta \sigma_{x y}\right) \rightarrow \infty$ [18]. In particular, even in homogeneous loading, ruptures will accelerate asymptotically to $C_{R}$ with an increasing length.

In our experiments, as we nucleate ruptures at $x \approx 0$, the medium is not infinite and the effect of plate edges should be taken into account. The exact dynamic solution for this geometry is a formidable task. We find, via finite element simulations (as described in Ref. [16]), that dynamic crack propagation in our geometry can still be well approximated by Eq. (2) when the value of $G_{\text {II }}^{S}$ for an infinite geometry is replaced by $G_{\mathrm{II}}^{S}\left(l, \Delta \sigma_{x y}\right)$ corresponding to an edge crack (Fig. S1 [21]).

To validate the equation of motion [Eq. (2)], we need to independently measure both $\Gamma$ and $G_{\mathrm{II}}^{S}$. $\Gamma$ is obtained as follows. The dynamically measured stresses are fitted with Eq. (1), where $K_{\text {II }}$ is the sole free parameter. The obtained values of $K_{\text {II }}$ are then related to $\Gamma[7,16]$ (see Ref. [21] for details). $\Gamma$ is roughly independent of $C_{f}$, and inhomogeneities of the system are reflected in the $\sim 40 \%$ variation of $\Gamma$ along the frictional interface (Fig. S3 [21]). $G_{\text {II }}^{S}\left(l, \Delta \sigma_{x y}\right)$ is calculated explicitly by using the measured $\Delta \sigma_{x y}$ [Fig. 2(a)], when $\Delta \sigma_{x y}$ values for small $l$ are extrapolated [21]. The corresponding profiles of $G_{\mathrm{II}}^{S}\left(l, \Delta \sigma_{x y}\right)$ and $\Gamma$ are shown in Fig. 2(c). We note that, while $\Gamma$ was obtained by the measurement of the dynamic singular fields (near fields) at the rupture tip, $G_{\mathrm{II}}^{S}$ was calculated using solely the stress drops $\Delta \sigma_{x y}$ ahead of the rupture tip (far fields).

We now compare our velocity measurements of frictional ruptures, shown in Fig. 2(b), with the dynamic predictions embodied in $g_{\mathrm{II}}\left(C_{f}\right)$. Figures 2(b) and 2(c) demonstrate that slow ruptures (blue line) propagate when the elastic energy released by a unit advance of the crack is nearly balanced by the dissipated energy, $G_{\mathrm{II}}^{S} \approx \Gamma$. Loading conditions that result in excess elastic energy, $G_{\mathrm{II}}^{S} \gg \Gamma$, generate a rapid acceleration to $C_{R}$ (green line).

Figure 2(d) shows that $C_{f}(l)$, when plotted with respect to $G_{\text {II }}^{S}\left(l, \Delta \sigma_{x y}\right) / \Gamma$, collapse to the single functional form predicted by Eq. (2). The applicability of the shear crack equation of motion to frictional ruptures is further
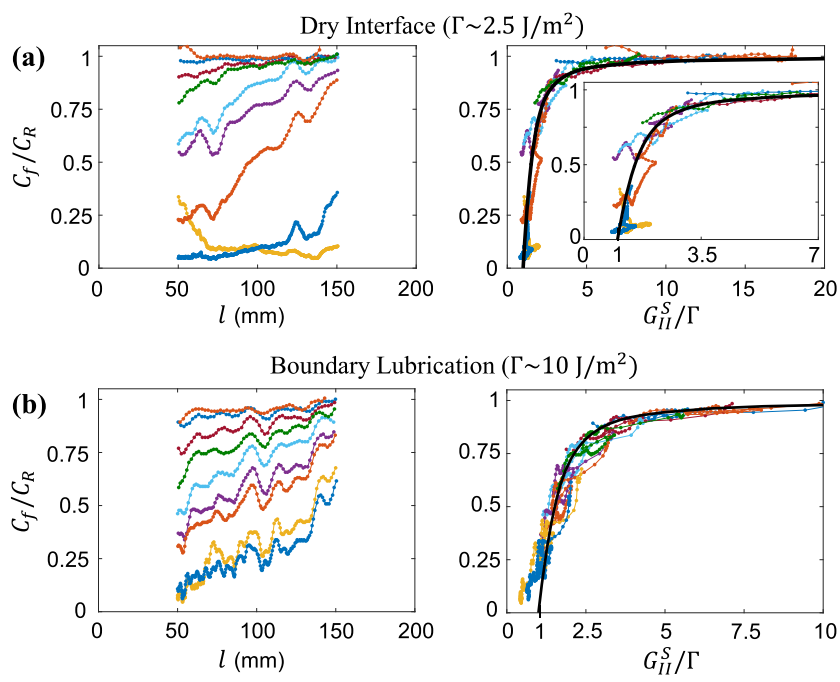

FIG. 3. Generality of the equation of motion. $C_{f}(l)$ profiles, in experiments with dry (a) and boundary-lubricated interfaces (b), having significantly different values of $\Gamma$ and spanning the whole sub-Rayleigh regime. All rupture events follow the LEFM prediction (black curves) given by energy balance [Eq. (2)] for brittle cracks (right).

demonstrated in Fig. 3(a), where we have supplemented the three typical events discussed in Fig. 2 with numerous other rupture events. These all had the same value of $\Gamma$ but were generated under numerous different applied stress profiles.

We demonstrate the generality of our results by further examining ruptures in the boundary-lubrication regime, which were recently shown [17] to be described by the same singular form [Eq. (1)] as for dry interfaces but with significantly larger $\Gamma$. Figure $3(\mathrm{~b})$ shows that ruptures propagating along lubricated interfaces also obey Eq. (2), in this case for a significantly larger $\Gamma$. Measurements of $\Gamma$ and $\Delta \sigma_{x y}$ profiles are provided in Figs. S3 and S4 [21].

Finally, we compare our experiments with the predictions of Eq. (2) at the limit of large $G_{\mathrm{II}}^{S} / \Gamma$, where $C_{f}$ asymptotically approaches $C_{R}$ and variations of $C_{f}$ cannot be experimentally distinguished. To this end, we make use of the evolution of the material velocity amplitudes, $\dot{u}_{x}(l)$ ( $u_{x}$ is the $x$ component of the displacement field), measured at $y=3.5 \mathrm{~mm}$ [7]. Figure 4 (left) presents $\dot{u}_{x}(l)$ measurements during the rupture events shown in Fig. 3. Near the crack tip, $\dot{u}_{x}$ is dominated by the universal singular form of the stress and strain fields. We compare measurements with different values of $\Gamma$ by normalizing $\dot{u}_{x}$ amplitudes by $\sqrt{\Gamma}$, following the scaling of the singular fields [17]. Figure 4 (right) demonstrates that all $\dot{u}_{x}(l) / \sqrt{\Gamma}$ collapse perfectly to the single curve, predicted by LEFM, as a function of $G_{\mathrm{II}}^{S}\left(l, \Delta \sigma_{x y}\right) / \Gamma$. At the limit of large $G_{\mathrm{II}}^{S} / \Gamma$, while $C_{f}$ asymptotically approaches $C_{R}$ (Fig. 3 ), $\dot{u}_{x}$ does not reach a finite limit but progressively increases. This unlimited growth of $\dot{u}_{x}$ is a direct consequence of the strain fields' 

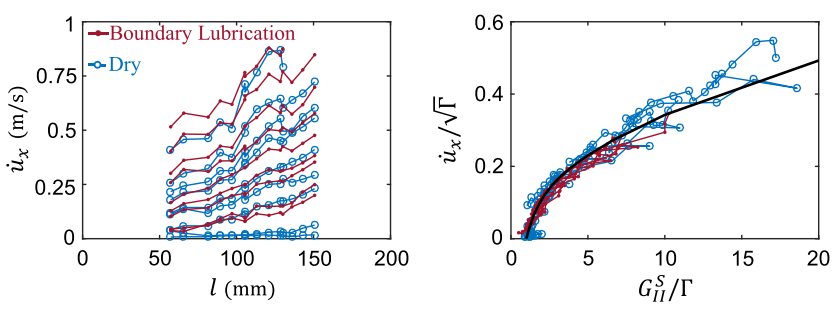

FIG. 4. Comparison to LEFM predictions for rupture velocities approaching $C_{R}$. (Left) Maximal material velocities $\dot{u}_{x}$ measured at points $(l, y=3.5 \mathrm{~mm})$ for the ruptures presented in Fig. 3 . (Right) Collapse of these measurements is obtained, as described by LEFM, when $\dot{u}_{x}$ amplitudes are plotted with respect to $G_{\mathrm{II}}^{S} / \Gamma$ and scaled by $\sqrt{\Gamma}$. Note that $\dot{u}_{x}$ amplitudes span 2 orders of magnitude and increase significantly as $C_{f} \rightarrow C_{R}$. The solid line designates the values predicted by LEFM. The values of $\dot{u}_{x}$ provide a more detailed comparison with LEFM than the equation of motion for $C_{f} \rightarrow C_{R}$ as occurs for $G_{\mathrm{II}}^{S} \gg \Gamma$.

divergence as $C_{f} \rightarrow C_{R}$ (see Fig. S3 [21] and Ref. [7]). This extreme sensitivity of $\dot{u}_{x}$ on $C_{f}$ enables us to provide a high-resolution quantitative comparison with the fracture mechanics theory [28] in the vicinity of $C_{R}$.

Our measurements have shown that the evolution of $C_{f}(l)$ is governed by energy balance, as determined by the LEFM singular fields [Eq. (2)]. Whereas a specific material (with both dry and boundary-lubricated interfaces) was considered in this study, the results are general so long as several necessary conditions are satisfied. First, a region in the rupture tip's vicinity should exist where $\sigma_{i j} \sim r^{-1 / 2}$. Typically, this condition is satisfied when the rupture length is large with respect to the process zone, the region where the stress singularity is regularized $(<3 \mathrm{~mm}$ in the current experiments; see Fig. S2 [21]). Furthermore, LEFM is applicable when, as is the case in our experiments, there is no significant variation of $\sigma_{x y}^{r}$ after the passage of the rupture tip. In this case, $\sigma_{x y}^{r}$ can be subtracted, due to linearity of the elastic equations, and frictional ruptures can be mapped to simple cracks with traction-free faces.

Some comments are in order. As Fig. 1(c) shows, there are slight variations of $\sigma_{x y}^{r}$ with $C_{f}$ (and therefore also with the slip velocity). These were taken into account in calculating $\Delta \sigma_{x y}(l)$. Predicting $C_{f}(l)$ based on the knowledge of $\Gamma$ and $\sigma_{x y}^{0}(l)$ alone, without the need for direct measurements of $\sigma_{x y}^{r}$, would be possible if a constitutive law for $\sigma_{x y}^{r}$ were formulated. Additionally, while in our experiments $\Gamma$ is roughly independent of $C_{f}$, the fracture theory can be applied to more complicated constitutive friction laws if these could be mapped to $\Gamma\left(C_{f}\right)[28,29]$. Finally, Eq. (2) can be used to predict $C_{f}(l)$ only if $G_{\text {II }}^{S}\left(l, \Delta \sigma_{x y}\right)$ can be explicitly calculated for the considered geometry. Our results are generic, at all times prior to the arrival of waves reflected from the sample's horizontal boundaries to the rupture tip [18]. In the case that we consider, the infinite medium approximation (with only the boundaries at $x=0$ taken into account) is valid. When the rupture strongly interacts with the boundaries, e.g., in a strip geometry, a different approach should be adopted [30], and, while energy balance should still be valid, a different equation of motion would be observed.

We have shown that the brittle fracture theory is in excellent quantitative agreement with our measurements for a large range of rupture velocities $0.04 C_{R}<C_{f}<C_{R}$ (ruptures that surpass $C_{S}[5,8]$ are beyond the scope of this work). Experimental observations [1,3,5-7] of the slow rupture regime, $C_{f} \ll C_{R}$, have drawn much attention, and their driving mechanism is still under debate. It has been suggested that slow ruptures emerge as a result of a crossover from a velocity weakening of friction at slow slip to velocity strengthening at higher slip rates [13-15]. Alternatively, slow ruptures have been observed in stochastic multiscale simulations [11]. While nontrivial friction laws might be a key mechanism for stabilizing slow ruptures, our current measurements suggest that slow ruptures may also emerge from any nontrivial stress distribution that would retain the "static" energy balance defined by $G_{\text {II }}^{S} \approx \Gamma$, along the propagation path. Such a scenario was also suggested in Ref. [31]. One could argue that Fig. 3(b) suggests that ruptures may also propagate even for $G_{\mathrm{II}}^{S}<\Gamma$. We believe that our measurement uncertainties preclude this conclusion; more precise measurements would be required.

Let us now consider rupture initiation. In the framework of brittle fracture, cracks lose their stability when they reach a critical length $l_{c}$ (the Griffith length), where $G_{\mathrm{II}}^{S}\left(l_{c}\right)=\Gamma$. While rupture propagation (Fig. 3) and arrest $[19,20]$ are dictated by the brittle fracture theory, the question of rupture nucleation is more subtle. For the Griffith criterion to apply, a singular crack should first exist. The spontaneous formation of a seed crack along the frictional interface which is needed for the nucleation process is an issue under debate [14,32-34]. In this work, the rupture nucleation was controlled by inducing a very slight perturbation of the out of plane stresses (mode III) at $x \approx 0$. It is conceivable that such an extreme sensitivity to local out of plane perturbations could be relevant to natural fault geometries with qualitatively similar aspect ratios (fault length/fault thickness).

This work was supported by the U.S.-Israel Binational Science Foundation (Grant No. 2016950), and Israel Science Foundation (Grant No. 1523/15) (all to I. S., E. B., and J. F.).

*Corresponding author. jay@mail.huji.ac.il

[1] S. M. Rubinstein, G. Cohen, and J. Fineberg, Nature (London) 430, 1005 (2004).

[2] P. G. Okubo and J. H. Dieterich, J. Geophys. Res. 89, 5817 (1984). 
[3] M. Ohnaka and L.-f. Shen, J. Geophys. Res. 104, 817 (1999).

[4] M. Mello, H. S. Bhat, and A. J. Rosakis, J. Mech. Phys. Solids 93, 153 (2016).

[5] O. Ben-David, G. Cohen, and J. Fineberg, Science 330, 211 (2010).

[6] S. Nielsen, J. Taddeucci, and S. Vinciguerra, Geophys. J. Int. 180, 697 (2010).

[7] I. Svetlizky and J. Fineberg, Nature (London) 509, 205 (2014).

[8] K. Xia, A. J. Rosakis, and H. Kanamori, Science 303, 1859 (2004).

[9] F. X. Passelègue, A. Schubnel, S. Nielsen, H. S. Bhat, and R. Madariaga, Science 340, 1208 (2013).

[10] M. Radiguet, D. S. Kammer, P. Gillet, and J.-F. Molinari, Phys. Rev. Lett. 111, 164302 (2013).

[11] J. K. Trømborg, H. A. Sveinsson, J. Scheibert, K. Thøgersen, D. S. Amundsen, and A. Malthe-Sørenssen, Proc. Natl. Acad. Sci. U.S.A. 111, 8764 (2014).

[12] Z. Peng and J. Gomberg, Nat. Geosci. 3, 599 (2010).

[13] Y. Bar-Sinai, E. A. Brener, and E. Bouchbinder, Geophys. Res. Lett. 39, n/a (2012).

[14] Y. Bar-Sinai, R. Spatschek, E. A. Brener, and E. Bouchbinder, Phys. Rev. E 88, 060403 (2013).

[15] B. M. Kaproth and C. Marone, Science 341, 1229 (2013).

[16] I. Svetlizky, D. Pino Muñoz, M. Radiguet, D. S. Kammer, J.-F. Molinari, and J. Fineberg, Proc. Natl. Acad. Sci. U.S.A. 113, 542 (2016).

[17] E. Bayart, I. Svetlizky, and J. Fineberg, Phys. Rev. Lett. 116, 194301 (2016).

[18] L. B. Freund, Dynamic Fracture Mechanics (Cambridge University, Cambridge, England, 1990).

[19] E. Bayart, I. Svetlizky, and J. Fineberg, Nat. Phys. 12, 166 (2016).
[20] D. S. Kammer, M. Radiguet, J.-P. Ampuero, and J.-F. Molinari, Tribol. Lett. 57, 23 (2015).

[21] See Supplemental Material at http://link.aps.org/ supplemental/10.1103/PhysRevLett.118.125501 for finite element simulations of mode II edge-crack propagation, and additional theoretical background and details of the experimental analysis. Included are Refs. [5,7,16,18, 22-27].

[22] H. Tada, P. C. Paris, and G. R. Irwin, The Stress Analysis of Cracks Handbook, 3rd ed. (American Society of Mechanical Engineers, New York, 2000).

[23] M. Aldam, Y. Bar-Sinai, I. Svetlizky, E. A. Brener, J. Fineberg, and E. Bouchbinder, Phys. Rev. X 6, 041023 (2016).

[24] A. C. Palmer and J. R. Rice, Proc. R. Soc. A 332, 527 (1973).

[25] A. N. B. Poliakov, R. Dmowska, and J. R. Rice, J. Geophys. Res. 107, ESE 6-1 (2002).

[26] O. Samudrala, Y. Huang, and A. J. Rosakis, J. Geophys. Res. 107, ESE 7-1 (2002).

[27] O. Ben-David and J. Fineberg, Phys. Rev. Lett. 106, 254301 (2011).

[28] A.-A. Gabriel, J.-P. Ampuero, L. A. Dalguer, and P. M. Mai, J. Geophys. Res. 117, n/a (2012).

[29] J.-P. Ampuero and Y. Ben-Zion, Geophys. J. Int. 173, 674 (2008).

[30] M. Marder, Phys. Rev. Lett. 66, 2484 (1991).

[31] Y. Kaneko and J.-P. Ampuero, Geophys. Res. Lett. 38, n/a (2011).

[32] K. Uenishi and J. R. Rice, J. Geophys. Res. 108, 2042 (2003).

[33] A. M. Rubin and J. Ampuero, J. Geophys. Res. 110, B11312 (2005).

[34] S. Latour, A. Schubnel, S. Nielsen, R. Madariaga, and S. Vinciguerra, Geophys. Res. Lett. 40, 5064 (2013). 